\title{
Relationship Between Radiographic Misfit and Clinical Outcomes in Immediately Loaded Complete-Arch Fixed Implant Supported Prostheses in Edentulous Patients
}

Robert Slauch

slauch@uchc.edu

\section{Recommended Citation}

Slauch, Robert, "Relationship Between Radiographic Misfit and Clinical Outcomes in Immediately Loaded Complete-Arch Fixed Implant Supported Prostheses in Edentulous Patients" (2019). Master's Theses. 1395.

https://opencommons.uconn.edu/gs_theses/1395 


\title{
Relationship Between Radiographic Misfit and Clinical Outcomes in Immediately Loaded Complete-Arch Fixed Implant Supported Prostheses in Edentulous Patients
}

\author{
Robert Walter Slauch, DDS \\ B.S., The Pennsylvania State University - 2011 \\ D.D.S., University of Maryland, Baltimore College of Dental Surgery - 2016
}

A Thesis Submitted in Partial Fulfillment of the

Requirements for the Degree of:

Master of Dental Science

at the University of Connecticut

2019 


\begin{abstract}
APPROVAL PAGE
Master of Dental Science Thesis
\end{abstract}

\title{
Relationship Between Radiographic Misfit and Clinical Outcomes in Immediately Loaded Complete-Arch Fixed Implant Supported Prostheses in Edentulous Patients
}

Presented by:

Robert Walter Slauch, D.D.S.

Major Advisor

Avinash Bidra, B.D.S., M.S.

Associate Advisor

John Agar, D.D.S., M.A.

Associate Advisor

Thomas Taylor, D.D.S., M.S.D.

Associate Advisor

Chia-Ling Kuo, Ph.D

University of Connecticut

2019 


\section{TABLE OF CONTENTS}

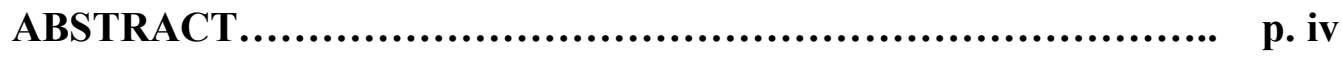

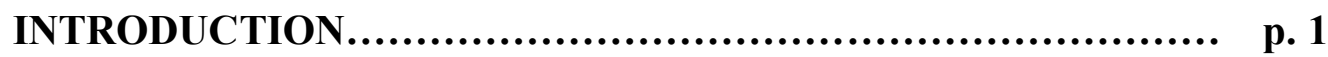

$\begin{array}{ll}\text { Literature Review } & \text { p. } 1\end{array}$

$\begin{array}{ll}\text { Rationale for the Study } & \text { p. } 7\end{array}$

OBJECTIVES AND HYPOTHESIS................................ p. 10

$\begin{array}{ll}\text { Research Objectives } & \text { p. } 10\end{array}$

$\begin{array}{ll}\text { Hypothesis } & \text { p. } 11\end{array}$

MATERIALS AND METHODS.................................... p. 12

$\begin{array}{ll}\text { Radiographic Misfit Selection } & \text { p. } 12\end{array}$

Radiographic Analysis p. 13

$\begin{array}{ll}\text { Statistical Analysis } & \text { p. } 14\end{array}$

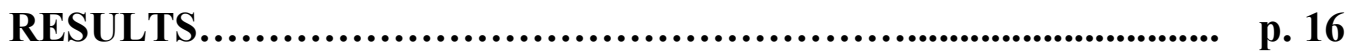

$\begin{array}{ll}\text { Prevalence of Misfit } & \text { p. } 16\end{array}$

Prevalence of Clinical Covariables $\quad$ p. 16

Early and Late Implant Complications p 18

Early and Late Prosthesis Complications p. 19

Nature of Misfit Resolution $\quad$ p. 19

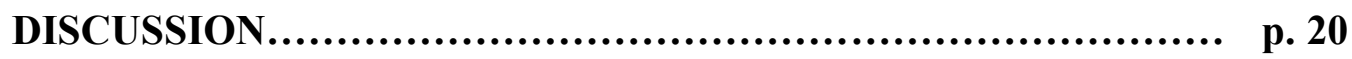

STUDY LIMITATIONS.............................................. p. 24

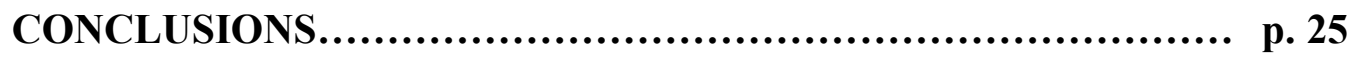

FUTURE RESEARCH.......................................... p. 26

REFERENCES................................................... p. 27

FIGURES AND TABLES............................................ p. 33 


\section{B. ABSTRACT}

STATEMENT OF PROBLEM: Dental literature has shown passive fit of dental prostheses is necessary to reduce strain on the implants or prosthetic components. However, misfit remains documented in the clinical setting. Previous authors have researched misfit by various methods including in-vitro, clinical, and radiographic analysis. However, their samples pertained to use of definitive single unit, fixed partial, and complete arch fixed implant supported prosthesis (CAFIP). To date, no research is present on the effect of uncorrected misfit on immediately loaded interim CAFIP.

PURPOSE: The purpose of this study is to examine if an uncorrected radiographically detectable immediate post-operative misfit (implant level or abutment level) in an immediately loaded interim CAFIP plays any role in early or late implant failure or prosthesis failures. In addition, clinical characteristics such as arch (maxilla/mandible), implant position (anterior/posterior), type of implant (alveolar/extra maxillary), implant orientation (tilted/straight), connection (external/internal), surgery type (free-hand/guided), and total number of implants per arch in relation to were analyzed.

MATERIALS AND METHODS: After obtaining IRB approval, immediate post-operative and 4-month panoramic radiographs of 425 arches (311 patients; 2,025 implants) with immediately loaded CAFIPs were analyzed in a retrospective chart review. The charts were reviewed to study the outcome of the implants and prostheses. Misfit and non-misfit implants within a given arch were summarized separately with respect to each clinical characteristic and the difference between misfit and non-misfit groups was tested using a mixed-effects logistic regression model with a patient-specific random intercept. An p-value of 0.05 will be used to reject any foreseeable null hypotheses related to comparison between survival rates and misfit. 
RESULTS: Out of 425 arches reviewed (2,025 implants), 48 misfits were found within 33 arches (23 patients) for a $2.4 \%$ prevalence rate. In the misfit sample, 2 implant failures were documented during the healing phase, yielding an early implant survival rate of $95.8 \%$ and late implant survival rate of $100 \%$. A total of 4 interim CAFIP fractured during the healing phase for early and late prosthesis survival rate was $87.8 \%$ and $100 \%$, respectfully. None of the clinical variables analyzed were significantly associated with the misfit status $(p<0.05)$.

CONCLUSION: The results from this study showed that a misfit in the immediate load scenario may not play a detrimental role in the implant survival but may affect survival of the interim CAFIP. Results from this study could aid in implant clinical decision making, about whether a misfit in an immediately loaded CAFIP should be corrected immediately. 


\section{INTRODUCTION}

\section{Review of Literature}

Dental implant therapy has evolved into a ubiquitous treatment modality to replace a complete set of dentition to the edentulous maxilla and mandible. Over the past 25 years, innovative protocols have allowed patients to receive dental implants and an interim fixed complete denture the same day. ${ }^{1,2}$ Advancements in implant design and treatment planning now allows a complete arch fixed implant supported prosthesis (CAFIP) to be anchored to as little as four implants ${ }^{3,4}$. Dental implants used for complete arch rehabilitation have demonstrated high survival rates up to the 20 years. ${ }^{5,6}$ According to a meta-analysis by Papaspyridakos et al, implants in the mandible had 98\% cumulative survival rate (CSR) at 10-year follow-up. ${ }^{5}$ Further, Lambert et al. reported $88 \%$ survival rate in the maxilla at 15 -year follow-up. ${ }^{6}$ The high survival rates can be attributed to the advancements of implant engineering and implant surface technology. ${ }^{7}$ Further, the splinting effect demonstrated with the attachment of an interim CAFIP during the three month healing phase has shown positive clinical benefit. ${ }^{8}$ However, implant survival is not $100 \%$ and can fail for a variety of reasons. Implant biomechanics for example, if neglected, can be an aggressive catalyst to biologic and prosthetic complications. Therefore, it is the responsibility of the practitioner to recognize the essential biomechanics involved with dental implant rehabilitations and the potential clinical challenges generated.

A frequently discussed topic in implant biomechanics is passive fit. Although there is no true consensus on a definition, ${ }^{9-11}$ for the purpose of this study it can be defined as "the adaptation of one component to another that does not impart strain." 12 A primary goal of a CAFIP is to achieve passive fit at the implant to prosthesis interface. This ensures an intimate 
adaptation at the implant-abutment junction which has been proposed to decrease the risk of screw-loosening, marginal bone loss and/or implant/abutment fracture. ${ }^{13}$

Consequently, passive fit is not always achieved due to prosthesis fabrication techniques and inherent material distortions and a horizontal or vertical gap between the implant to prosthesis interface can occur. This clinical outcome has been termed "misfit" and two types, horizontal and vertical, have been described. Horizontal misfit is a misfit in the mesial-distal or buccal-lingual direction and vertical misfit in coronal-apical direction.

The significance of a misfit at the implant-prosthesis junction in osseointegrated implants has been extensively debated in the literature. Historically, a gap at the interface was considered a normal occurrence up to $150 \mu \mathrm{m} .{ }^{14}$ However, the initial research was in an era where the lostwax technique and metal casting of complete arch frameworks were employed. These procedures have been proven to be technique sensitive and produce varying amounts of distortion. ${ }^{15}$ With the advent of $\mathrm{CAD} / \mathrm{CAM}$ technology, prosthetic components are now adapting at a more intimate level, re-igniting the debate about marginal gaps at the implant interface and its potential clinical consequences. According to Jemt et al. there is no significant difference in passive fit vs nonpassive fit at the implant-prosthesis interface. Further, Jemt proposed a certain biologic tolerance to misfit. ${ }^{16}$ However, this tolerance should not be compared to the forbearance provided by the periodontal ligament in natural dentition. ${ }^{17}$

Various methods implemented to assess misfit include in-vitro, intra-oral and radiographic analyses. Scanning electron microscopy can be used in the laboratory to examine the precision-fit of prosthetic components. An in-vitro study by Katsoulis et al. concluded photogrammetric and laser scanners in conjunction with CAD/CAM technology produces a statistically significant smaller micro-gap compared to traditional soldered frameworks. ${ }^{18}$ Intra- 
oral assessments include the use of visual examination, disclosing materials, perceptual feedback of the interface with a titanium or plastic dental explorer, and tactile feedback. ${ }^{13}$ Dental periapical and panoramic radiographs can also be used to assess prosthetic adaptation. ${ }^{19-21}$ Further, the one-screw test is an alternative method verify passive fit. In this test, the practitioner screws the definitive CAFIP framework cast with only one screw in the most distal implant abutment. Passive seating is then verified visually at other implant sites by complete seating at the implant-prosthesis interface. ${ }^{22}$ Finally, the screw resistance test can be utilized. Screws are tightened in the abutments closest to the midline first, then one-by-one moving distally until resistance to tightening is felt. A misfit was considered when more than one-half turn was needed to fully tighten the screw. ${ }^{14}$

Dental radiography to verify prosthetic components is not novel to the dental literature. It has been the standard of care for decades to assess marginal adaptation of tooth-borne crowns, inlays, onlays, etc. ${ }^{23,24}$ It allows the practitioner to examine components that cannot be verified clinically through visual or tactile feedback. In terms of complete arch rehabilitation, the panoramic radiograph is the standard radiograph exposed to very adequate clinical outcomes. It has advantages over intra-oral radiographs since the entire maxilla and mandible can be visualized. Adjacent anatomic sites such as the mandibular canal/foramina and maxillary sinuses can be identified to allow proper implant selection and placement. ${ }^{25}$ In addition, due to bone reduction procedures that alter the anatomy of a patient's jaw with respect to the adjacent structures, conventional periapical radiographs are often impossible to take. A panoramic radiograph is readily taken post-operatively to assess final implant orientation and complete seating at the implant-abutment junction. Most practitioners also expose a three to four month follow up radiograph to re-examine the sub-crestal and supra-crestal prosthetic components. 
It should be noted that definitive single unit and fixed-partial implant supported restorations render the ability to be cement retained or screw retained. A few studies have demonstrated that cement retained restorations have less strain compared to screw retained, due to the potential tolerance related to the cement gap. ${ }^{26,27}$ Yet, if the implant is placed in a prosthetically favorable position, the decision to use cement versus screw retained is subjective amongst practitioners. However, CAFIPs are most often screw retained due to the need of retrieval for potential future adjustments. Thus, the potential consequences of misfit and increased strain must be recognized.

Presently, the majority of literature on misfit explores biological or prosthetic complications in definitive single unit, fixed-partial and complete arch frameworks on osseointegrated implants ${ }^{14-20}$. However, research on the clinical implications (implant/prosthesis survival rates) of uncorrected misfit in immediately loaded (interim) all-acrylic resin CAFIP in freshly placed implants has not been reported. This information has imperative value to the dental community for a variety of reasons described below.

Compared to definitive full arch prostheses, interim prostheses lack a metal framework and are typically fabricated of poly-methyl methacrylate (PMMA) due to simplicity, ease of adjustments and reduced cost to the patient and clinician. ${ }^{2}$ However, the all-acrylic resin may undergo flexure with time due to the inherent nature of the material and may fracture. Additionally, inadequate prosthetic space can render it less bulky and may increase risk for fracture. A study by Drago reported 18\% of 191 interim CAFIPs needed repair. Seventy percent of the repairs were fractures of the denture base at the implant/prosthetic junction. ${ }^{28}$ Further, the fracture rate increases if prosthetic height guidelines are violated. ${ }^{29,30}$ With the advent of 
CAD/CAM, monolithic PMMA interim prosthesis are fabricated with increased strength properties, but repairs are still necessary.

Monolithic PMMA CAFIPs are typically fabricated prior to flapless guided surgery with the use of $\mathrm{CAD} / \mathrm{CAM}$ technology. The pre-fabrication has reduced surgery and prosthetic chairside time for the patient and practitioner. However, misfit in pre-fabricated immediately loaded CAFIPs has been documented in the literature. In a study by Landázuri-Del Barrio et al., 13 of 16 patients who received flapless guided surgery and an immediately loaded monolithic PMMA CAFIP demonstrated misfit between the abutment and temporary cylinder. Due to this finding, the authors argued pre-fabricated monolithic CAFIPs have clinical drawbacks and the conversion prosthesis should be created based on the precise orientation of the implants at time of surgery. ${ }^{31}$ In addition, a study Yilmaz et al. showed adjustments with laser welding was typically needed for immediate metal-resin CAFIPs to correct misfit. ${ }^{32}$ To offset misfit, specific guided abutments were fabricated to allow compensation of vertical misfit up to $0.4 \mathrm{~mm}$ at the abutment-prosthesis interface. These self-adjusting abutments are typically used to mitigate thick soft tissues present in the flapless surgery protocol. ${ }^{33}$

Misfit in interim CAFIPs may place unwanted strain on the corresponding implants, abutments, and prosthetic components leading to an increased risk of fracture mentioned above. Further, the increased strain along with corresponding prosthesis fracture may induce enough stress to cause marginal bone loss, implant fracture, abutment fracture and eventually implant failure. Implant failure can be defined as complete loss of osseointegration leading to loss of stability and removal of the implant. To date, these theories about misfit and implant failure have not been proven and are considered dogma in the dental community. Therefore, there is clinical 
benefit to determine if misfit at the implant interface plays a significant role in increasing prosthetic complications and potential implant failure rates in the immediately loaded CAFIP.

It can be argued that follow-up radiographs can typically demonstrate three methods of clinical misfit resolution. First, the misfit auto-corrected before any clinical intervention by the practitioner. Second, the misfit was clinician corrected by hand tightening of the prosthetic screw. Third, the misfit was never clinically corrected and the open gap was still present. To date, no studies examine the clinical effects of these scenarios. Therefore, it is imperative to determine if implant or prosthesis survival rates in immediately loaded conditions were significantly affected by the different methods of resolution.

Early literature showed it was necessary to delay implant loading three to six months for complete osseointegration. ${ }^{33-35}$ As years progressed, loading times have decreased and immediate loading gained popularity in the dental community. A 2005 study by Attard and Zarb demonstrated its popularity and success depending on systemic and anatomic determinants. ${ }^{36}$ Additional authors began to explore the changes in implant stability during critical three month healing phase with resonance frequency analysis (RFA). ${ }^{37,38}$ In a study by Balshi et al., RFA demonstrated a decrease in bone-implant stability the first month after placement, followed by an increase in stability the second and third months. ${ }^{39}$ This suggests adaptive osteoblastic and osteoclastic activity around the implant during the critical healing period. Unwanted strain on dental implants during this healing period should be limited. It has been proposed that a misfit between the implant and an immediately loaded CAFIP could impose strain that disrupts implant stability, and potentially lead to early implant failure. Therefore, further research on misfit is warranted. 


\section{Rationale for the Study}

Dental implant therapy has become a mainstream approach to provide the necessary subcrestal anchorage for complete arch prosthetic therapy in the edentulous maxilla and mandible. In addition, immediate implant loading has drastically revolutionized the field of implant prosthodontics allowing patients to receive fixed dentition the same day of surgery. In the past 10 years, the All-on-Four ${ }^{\mathrm{TM}}$ treatment concept has streamlined rehabilitation of the edentulous maxilla and mandible. ${ }^{3,4}$ The simplicity of the protocol due to the graftless modality can allow a novice and/or experienced practitioner to use the procedure and achieve similar implant and prosthetic survival rates. A study by Balshi et al. retrospectively examined 800 implants in the All-on-Four ${ }^{\mathrm{TM}}$ protocol. Three-hundred implants in the edentulous maxilla and 500 implants in the mandible had a CSR's of $97.8 \%$ and $96.3 \%$, respectively. ${ }^{40}$ A larger study by Niedermaier et al. analyzed 2,081 implants attached to immediate CAFIPs and had a CSR of $97.0 \% .{ }^{41}$ Due to ease and predictability, the protocol is becoming very popular as trends in edentulism increase.

According to Douglass et al., the edentulous population is declining at a rate of $30 \%$ over the last 30 years. However, due to the increasing "baby-boomer" population trends in complete edentulism is expected to increase from 33.6 million in 1991 to 37.9 million in $2020 .{ }^{42}$ Rehabilitation with removable complete dentures is declining as more patients are electing oral rehabilitation with dental implants and a CAFIP. Further, both dentists and prosthodontists are including procedures like the All-on-Four ${ }^{\mathrm{TM}}$ protocol in their clinical arsenal at astounding rates. Due to the increase demand of this protocol from both patent and practitioner, it is imperative for clinicians to be knowledgeable about how to manage potential biomechanical complications of implant supported complete arch rehabilitations. If neglected, unfavorable biomechanics can have detrimental effects to both implant and prosthetic survival. 
When attaching an interim CAFIP, it is the goal to achieve passive fit to all implants in the arch. Passive seating insures the elimination of unnecessary biomechanical strain at the implant to prosthesis interface. In some circumstances, passive fit is not achieved and a vertical or horizontal misfit at the implant abutment interface can occur.

As mentioned previously, interim CAFIPs typically lack a metal framework and are typically fabricated of poly-methyl methacrylate due to simplicity, ease of adjustments and reduced cost to the patient and clinician. ${ }^{2}$ In addition, the all-acrylic resin used to engage implants to temporary cylinders during the conversion protocol may undergo polymerization shrinkage. This polymerization shrinkage may cause passive fit to be uncontrollable at the implant-prosthesis interface, resulting in potential misfit. With the advent of CAD/CAM, guided surgery, and computer-generated surgical templates, monolithic PMMA interim prostheses are fabricated with increased accuracy at the implant-abutment junction. However, not all complete arch reconstructions have the ability to be performed with guided surgery due to anatomic and clinical constraints. Additionally, increased soft tissue thickness when not carefully managed, may allow soft tissue entrapment between the abutment and prosthesis to result in a misfit.

When used in a flapless scenario, monolithic PMMA CAFIPs have documented misfit in the dental literature. In a study by Landazuri-Del Barrio et al., 13 of 16 patients who received flapless guided surgery and an immediately loaded monolithic PMMA CAFIP demonstrated misfit between the abutment and temporary cylinder. The authors claimed pre-fabricated monolithic CAFIPs have clinical drawbacks and the conversion prosthesis should be created based on the precise orientation of the implants at time of surgery. ${ }^{31}$ In addition, a study Yilmaz et al. showed adjustments with laser welding was typically needed for immediate metal-resin CAFIPs to correct misfit. ${ }^{32}$ Implant companies have attempted to offset misfit in the flapless 
scenario by creating specific guided abutments that allow compensation of vertical misfit up to $0.4 \mathrm{~mm}$ at the abutment-prosthesis interface. These self-adjusting abutments are typically used to mollify thick soft tissues present in the flapless surgery protocol. ${ }^{33}$

To date, there is no literature that has examined the clinical outcomes of uncorrected vertical misfit in the immediate load scenario. Current dental literature on vertical and horizontal misfit only studies clinical complications in definitive single unit, fixed-partial, and complete arch frameworks, where osseointegration is typically complete at this point. It is the first three months post-placement where the implants are undergoing the most biological changes and implant stability is often changing. It is during this critical healing period where the immediately loaded complete arch interim prostheses are in function and misfit is probable. Research examining misfit in the immediate loading scenario has immense benefits due to the rising popularity of the procedure.

Therefore, this retrospective study seeks to determine if an uncorrected post-operative misfit plays a significant role in early and/or late implant or prosthesis survival rates. Results from this study can help practitioners determine the clinical importance of misfit in immediately loaded CAFIPs and if an uncorrected open-gap is detrimental to the overall complete arch rehabilitation. 


\section{OBJECTIVES AND HYPOTHESIS}

\section{Research Objectives}

This study employed the use of panoramic radiography and patient chart note records to examine the following characteristics of 425 edentulous jaws in order to know:

- The prevalence rate of radiographic misfit (horizontal or vertical) in immediately loaded all-acrylic resin complete arch fixed implant supported prostheses (CAFIPs).

- The rate of early or late implant failure in treatments associated with misfit in immediately loaded all-acrylic resin CAFIPs.

- The rate of early or late prosthesis failure in treatments associated with misfit in immediately loaded all-acrylic resin CAFIPs.

- If there are differences in the nature of misfit resolution:

- Spontaneous resolution without clinical intervention

- No resolution of the misfit, without clinical intervention

- Clinical intervention to close the implant-to-prosthesis interface

○ No resolution of misfit

- If clinical characteristics such as arch (maxilla/mandible), implant position (anterior/posterior), type of implant (alveolar/extra maxillary), implant orientation (tilted/straight), connection (external/internal), surgery type (free-hand/guided), and total number of implants per arch play any significant roles with misfit. 


\section{Hypothesis}

This study was a retrospective cohort study on edentulous patients examining patient chart records, to determine if an uncorrected post-operative open gap (radiographic misfit) in an immediately loaded complete arch fixed implant supported prosthesis has a relationship with early or late implant or prosthesis failures.

The null hypotheses for this study were as follows:

There is no difference between:

1. the prevalence rate of radiographic misfit (horizontal or vertical) in immediately loaded all-acrylic resin CAFIPs.

2. the rate of early or late implant failure in treatments associated with misfit in immediately loaded all-acrylic resin CAFIPs.

3. the rate of early or late prosthesis failure in treatments associated with misfit in immediately loaded all-acrylic resin CAFIPs.

4. the nature of misfit resolution.

5. the covariates, such as arch (maxilla/mandible), implant position (anterior/posterior), type of implant (alveolar/extra maxillary), implant orientation (tilted/straight), connection (external/internal), surgery type (free-hand/guided), and total number of implants per arch. 


\section{MATERIALS AND METHODS}

\section{Radiographic Misfit Selection}

University of Connecticut Health Center Institutional Review Board approval was obtained and was granted exemption, as non-identifiable data was examined and collected in this study (Project number: UCHC-17-168-3.1). A total of 425 jaws were examined with post-operative and 3-4 month follow-up panoramic radiographs from patient records of 2 co-investigator's private practices (Dr. Glenn J. Wolfinger, Pi Dental Center, Ft. Washington, PA and Dr. Avinash Bidra, Cloverleaf Dental Center, Meriden, CT). All radiographs were de-identified for all purposes in the study.

The inclusion criteria for selecting a panoramic radiograph were as follows:

- A treatment where compete arch implant supported rehabilitation was performed with immediate loading of one or both jaws.

- The availability of an immediate post-treatment panoramic radiograph.

- The availability of a follow-up panoramic radiograph within three to six months postsurgery.

- A recognizable misfit at the implant-prosthesis interface that has not been immediately corrected by clinical intervention.

- The implant-prosthesis interface must be clearly visualized.

- Images must be of adequate diagnostic quality.

The exclusion criteria for eliminating a selected panoramic radiograph were as follows:

- Any panoramic radiograph did not satisfy any of the requirements listed in the inclusion criteria.

- Any panoramic radiograph where the conversion prosthesis was not immediately loaded. 
- A panoramic radiograph that demonstrates no misfit and full closure all implantprosthesis interfaces within the arch of interest.

- A panoramic radiograph with a poor resolution that does not allow for proper visualization and proper analysis.

- A panoramic radiograph that demonstrates partial arch rehabilitation.

\section{Radiographic Analysis}

Panoramic radiographs that were identified for use in the study had all personal identifiers removed from the digital image prior to analysis. Demographic information including age and gender were recorded for analysis. In addition, clinical characteristics such as type of arch (maxilla/mandible), implant position (anterior/posterior), type of implant (alveolar/extra maxillary), implant orientation (tilted/straight), connection (external/internal), surgery type (freehand/guided) were examined. The selected panoramic radiographs that fit the inclusion criteria and corresponding covariate data were copied to an encrypted and passcode protected external hard drive for use by the sole evaluator. Analysis was completed as followed:

- Analysis of immediate post-operative panoramic radiographs of patients with immediately loaded all-acrylic resin CAFIPs, to observe misfit (horizontal or vertical) between prosthesis and abutment/implant (Figure 1).

- Analysis of three or four-month post-operative panoramic radiographs (Figure 2) and/or definitive prosthesis panoramic radiographs of the same patients to observe the status of misfit and if resolution occurred (Figure 3).

- Analysis of the type of misfit observed (Figure 4,5,6). 
- Assessment of the identified radiographic misfits with the principle investigator to determine accuracy.

- Thorough analysis of clinical notes and radiographs to determine if there was clinical intervention to correct the misfit.

- Explore patient chart records and implant database systems to determine if any implant or prosthesis failures occurred and at what point in time (early or late).

- Assessment of implant database systems to gather important data such as surgery dates, failure dates, etc.

- Analysis of implant database systems to gather information on the desired covariates in this study.

- Conduct statistical tests to determine significance of misfit with analyzed variables.

\section{Statistical Analysis}

Data subjected to statistical analysis:

- Percent prevalence of misfit in immediately loaded CAFIPs.

- Percent prevalence of various types of misfit resolution or no resolution in immediately loaded CAFIPs.

- Early survival rate of implants when misfit was not corrected clinically on the day of insertion.

- Late survival rate of implants when misfit was not corrected clinically on the day of insertion.

- Early prosthesis survival rate when misfit was not corrected clinically on the day of insertion. 
- Late prosthesis survival rate when misfit was not corrected clinically on the day of insertion.

- Covariates such as arch (maxilla/mandible), implant position (anterior/posterior), type of implant (alveolar/extra maxillary), implant orientation (tilted/straight), connection (external/internal), surgery type (free-hand/guided), and total number of implants per arch played a significant role in misfit.

- The nature of misfit resolution.

All data were recorded in Microsoft Excel data sheets before statistical analysis. All of the statistical analyses were performed with the statistical software R 3.5.1 (R Core Team (2018). R: A language and environment for statistical computing. R Foundation for Statistical Computing, Vienna, Austria. URL: http://www.R-project.org/).

First, the implant data were fragmented into the categorical variables. These variables were summarized with frequencies and percentages. Misfit and non-misfit implants within a given arch were summarized separately with respect to each clinical characteristic and the difference between misfit and non-misfit groups was tested using a mixed-effects logistic regression model with a patient-specific random intercept. An p-value of $<0.05$ was chosen to reject any foreseeable null hypotheses related to comparison between survival rates and misfit. 


\section{RESULTS}

This study sought to examine the prevalence of early and or late implant and prosthesis failures when an implant or abutment level misfit was uncorrected in an immediately loaded CAFIP. This study also examined various clinical covariates to see if they play a significant role with misfits in the immediately load scenario. This study examined 425 jaws for a total of 2,025 implants in 311 patients (Table 1).

\section{Prevalence of Misfit}

Out of 2,025 implants, 48 implants were associated with misfit of the interim CAFIP within 33 arches in total of 27 patients (Table 1). The overall incidence misfit for complete arch conversion prostheses in the immediate load scenario was $2.4 \%$. In the 33 arches that met the inclusion criteria, the combined misfit and non-misfit implants from all arches equated to 182 samples (48 misfit, 134 non-misfit implants) (Table 1).

\section{Demographic Data}

This study recorded basic demographic data such as age and gender to understand the rage of subjects within the study. The overall age range spanned from 32 to 89 years old. Patient age found to have misfit ranged from 39 to 89 years old (Table 1). The gender distribution for misfit was $58 \%$ female and $42 \%$ male (Table 2 ).

\section{Clinical Characteristics of the analyzed data}

$\underline{\text { Arch Type }}$

Analysis of the incidence of misfit in the maxilla and mandible showed a higher rate in the maxilla. Thirty-six misfits (75\%) were found in the maxilla and 12 misfits in the mandible 
$(25 \%)$. Even though the maxilla had a higher incidence, the difference was not statistically significant (p-value: 0.876) (Table 2, Figure 7).

\section{$\underline{\text { Implant Position }}$}

Misfits were analyzed by region in the dental arch (anterior or posterior). Twenty-one misfits were found in the anterior region (44\%) and 27 misfits in the posterior region (56\%). When compared to the non-misfit implants, results were statistically insignificant ( $p$-value: 0.549) (Table 2, Figure 8).

\section{Type of Implant}

Implants placed in extra-maxillary bone (zygomatic and pterygomaxillary region) were analyzed and compared to implants placed in alveolar bone. Thirteen implants in extra-maxillary bone displayed misfit (27\%) and 35 implants in alveolar bone (73\%). However, it should be noted that only $26 \%$ of the total data was extra-maxillary implants $(n=48 / 182)$. The results were not statistically significant (p-value: 0.897 ) (Table 2, Figure 9).

\section{Implant Orientation}

The incidence of tilted implants was analyzed for misfit in relation to axially placed implants. Twenty-seven tilted implants had misfit (56\%) and 21 axial implants (44\%). The comparison with non-misfit implants within the same arch revealed statistically insignificant results (p-value: 0.814) (Table 2, Figure 10).

\section{Implant to Abutment Connection}

External versus internal connection of the implant to the abutment was analyzed. Fortysix misfits were found at the abutment-temporary coping level (96\%). Only two misfits at the implant-abutment level were found (4\%). However, the sample size for implant-to-abutment was 
low, accounting for only $2 \%$ of the total sample size $(n=3 / 179)$. The results were not statistically significant (p-value: 0.156)(Table 2, Figure 11).

\section{Surgery Type}

Differences in guided surgery versus free-hand surgery were observed. Forty-two misfits were found when free-hand surgery was performed (88\%). Only 6 misfits $(12 \%)$ when guided surgery was chosen. However, free-hand surgery did account for $88 \%$ of the total data $(\mathrm{N}=154 / 179)$. The results were statistically insignificant (p-value: 0.520) (Table 2, Figure 12).

\section{Total Number of Implants Per Arch}

With arches that had misfit, the total number of implants in a given arch ranged from 4 to 9 implants for an average of $5.76 \pm 1.58$. The mode was 6 implants per arch. The number of implants per arch was found to be statistically insignificant (p-value: 0.431)(Table 2).

\section{Early and Late Implant Complications}

Out of 48 misfits found, 2 implants failed in the 3-4-month healing phase (Table 2). No implants failed after the definitive prosthesis was inserted. This equated to a $96 \%$ early implant survival rate and $100 \%$ late survival rate, respectfully. Both failures occurred in the maxillary arch. The first failure was an alveolar, axially placed implant in the posterior region of the maxilla. A free-hand surgical protocol was used. The implant had an internal connection and the misfit was at the abutment to temporary coping level. Six implants were placed the arch. At the time of implant failure, another implant was place in an adjacent site and immediately loaded within the interim CAFIP. (Describe rescue)

The second failure was an extra-maxillary (pterygoid), tilted implant in the posterior region of the maxilla. A free-hand surgical protocol was used. The implant had an external 
connection and the misfit was at the abutment to temporary coping level. Six implants were placed in the arch. The failed implant site was ignored and another implant was not placed.

\section{Early and Late Prosthetic Complications}

Out of the 33 arches with misfit prostheses), 4 arches experienced fractures of the interim CAFIPs during the 3-to-4-month healing phase. Each CAFIP only fractured once. The prostheses were were repaired with acrylic resin material. After repair, no further complications with the prostheses resulted during the healing phase. There were no prosthetic complications after delivery of the definitive prostheses. This equated to $87.8 \%$ early prosthesis survival rate and a $100 \%$ late prosthesis survival rate. (Table 1) Three of the four interim CAFIPs had one misfit associated with them and one prostheses had a total of two misfits. This equated a total of 5 misfits involved in 4 fractured interim CAFIPs (10.4\%). (Table 2).

\section{Nature of Misfit Resolution}

The nature of misfit resolution was also tracked during the healing phase. Two misfits identified with misfit appeared to "auto-correct" during the healing phase (4\%). This "phenomenon" was observed when comparing the post-operative to the three-month panoramic

radiograph. The clinician mentioned there was no clinical intervention to fix these implants. The remaining $92 \%(n=44)$ of the implants were found to have corrected with the definitive prosthesis and $4 \%$ of the implants were lost to failure $(n=2)$. 


\section{DISCUSSION}

The purpose of this retrospective study was to determine if an uncorrected radiographically detected immediate post-operative misfit plays any role in early and/or late implant or prosthesis survival rates in immediately loaded complete arch fixed dental implant rehabilitations. In addition, various clinical co-variates were analyzed to test any significant relation to post-operative misfit. The majority of current literature on misfit explores biological or prosthetic complications in definitive single unit, fixed-partial and complete arch frameworks on osseointegrated implants ${ }^{14-20}$. To date, research on the clinical implications (implant/prosthesis survival rates) of uncorrected misfit in immediately loaded (interim) allacrylic resin interim CAFIPs in freshly placed implants has not been reported. This study sought to explore the importance of misfit in the immediate load scenario and if an uncorrected misfit is determinantal to the overall implant rehabilitation.

A total of 2025 implants in 425 arches were analyzed. The overall incidence of misfit in the complete arch immediate load scenario was determined to be very low at $2.4 \%$. This low incidence is a significant and promising finding of this study. A total of 48 samples of misfit were discovered and 2 implant failures associated with misfit occurred. This corresponded to and early and late implant survival rate was $96 \%$ and $100 \%$, respectively.

Successful osseointegration of dental implants is multifactorial process. However, in the compete arch scenario, perhaps the most important aspect is primary stability. In a systematic review by Papaspyridakos et al., primary stability was cited as the most important factor for immediate load determination in full arch surgeries ${ }^{45}$. Further, the splinting effect demonstrated with the attachment of an interim complete arch fixed prosthesis during the three-month healing phase has shown positive clinical benefit ${ }^{8}$. From the results of this study, it can be suggested that 
misfit does not play a detrimental role in early implant survival as long as primary stability is achieved at the time of surgery and the cross-arch splinting effect is achieved with the interim all-acrylic prosthesis.

Four of the 33 arches where misfit was present experienced a fracture of the interim CAFIP during the three-to-four-month healing period. This correlated to a $12.2 \%$ early prosthesis complication rate. A 2016 study by Drago examined the frequency and type of prosthetic complications in immediately loaded CAFIPs. His study reported and $18 \%$ of 191 CAFIPs needing repair. ${ }^{28}$ Further, the fracture rate increased if prosthetic height guidelines were violated. ${ }^{29,30}$ In the present study, the early prosthesis complication rate of was $6 \%$ less, even when this study knew there was a post-operative mechanical complication (misfit) involved within that given arch. There was no mention of pre-determined misfit in the Drago study. As stated previously, interim prostheses lack a metal reinforcement framework and are typically fabricated of poly-methyl methacrylate due to simplicity, ease of adjustments and reduced cost to the patient and clinician. ${ }^{2}$ However, the all-acrylic resin may undergo flexure with time due to the inherent nature of the material and may fracture. Similar complication rates are present in the literature ${ }^{28}$ when misfit was not present, unlike the present study. Therefore, the results from this study suggest misfit may contribute to the survival of interim CAFIPs but the overall complication of interim CAFIP fracture is multifactorial.

A possible reason more implant prosthesis complications were not observed could be due to biological tolerance to misfit as described by Jemt et al. ${ }^{16}$ However, current observations point to strict adherence of proper surgical and prosthetic protocols for complete-arch dental implant rehabilitations. 
A 2017 study by Drago ${ }^{46}$ analyzed cantilever lengths and anterior-poster spread ratios (CL/AP) of interim all-acrylic resin CAFIPs to their relationship with prosthetic complications. The study found a (CL/AP) of 0.5 to 0.6 generally resulted in successful interim prostheses while in function. The present study did not measure cantilever lengths. However, there was careful attention by the practitioners in this study to have the cantilever length in all interim CAFIPs not exceed one-half tooth length distal from the terminal abutment.

Another observation is adequate strength and bulk of the interim CAFIP. Proper space analysis and subsequent bone reduction is necessary to gain the prosthetic space required for CAFIPs. ${ }^{47}$ If ignored, prosthetic and esthetic complications can occur. Proper occlusion of the interim CAFIPs is also imperative. The occlusal scheme for CAFIPs takes its concepts from classic fixed prosthodontics principles. A mutually protected occlusion at an acceptable vertical dimension devoid of excursive interferences is desired. This will inhibit unnecessary strain on the interim CAFIP. With the low incidence of implant failure in this study, one could argue if complete passive fit is even needed for osseointegration, if surgical and prosthetic guidelines are respected.

The nature of misfit resolution was another variable analyzed in this study. Two implants (4\%) were observed to have auto-corrected during the 3-4-month healing phase without any clinical intervention. There are biologic and prosthetic hypotheses as to why this may have occurred.

From a biologic perspective, bone adaptation may be occurring around the implant during the healing phase. Any strain caused by a misfit could potentially influence the osteoclastic/osteoblastic turnover as the implant loses primary stability and enters the secondary stability phase. In addition, the presence of soft tissue edema or hypertrophic tissues overlying 
the implant or abutment interface at the time of flap closure could inhibit complete seating of the interim prostheses. As the edema resolves and/or necrosis of the soft tissues initially causing entrapment, the prosthesis may be able to full seat.

Finally, dental arch, implant position, type of implant, implant orientation connection, surgery type, and total number of implants per arch were all determined to be statistically insignificant in relation to misfits in the immediate load scenario. It was hypothesized that a greater number of implants per arch would produce more misfits. The rationale is based upon more implant/abutment or abutment/prosthesis interfaces per arch, the more change for misfit. However, the data was determined to be insignificant. 


\section{STUDY LIMITATIONS}

The nature of this study included the following limitations:

- Use of panoramic radiographs: Although the panoramic radiograph is the standard radiograph exposed in the complete arch rehabilitation, there are issues with standardization and distortion between each exposure. However, panoramic radiographic assessment has been previously used in the literature to assess misfit. $^{25}$

- The availability of 3-4-month panoramic radiographs for each sample. In these instances, the final radiograph was used to determine misfit resolution.

- Presence of artifact in the panoramic radiographs which could have produced false negatives and/or false positives.

- All observations were made by 1 observer, which may introduce bias in the data collection. However, it can be argued it eliminated heterogeneity. 


\section{CONLCUSIONS}

Rehabilitation of the edentulous maxilla and mandible with complete arch fixed implant supported prostheses has become increasing popular in the field of prosthodontics. The advent of immediately loading an interim CAFIP the same day of surgery has broken the historical barriers that delayed loading was always necessary for implant survival. Thus, it is of benefit to the dental community to examine any consequences of uncorrected misfit in immediately loaded CAFIPs and assess its overall impact to the dental rehabilitation from a clinical as well as a medico-legal perspective. Baseline data from this study can help practitioners determine the clinical importance of misfit in immediately loaded CAFIPs.

Based on the results of this retrospective study, the following conclusions were drawn:

1. The overall prevalence of radiographically detectable misfit in immediately loaded complete arch all-acrylic interim prosthesis was $2.4 \%$

2. The early and late implant survival rate in implants that demonstrated misfit was $96 \%$ and $100 \%$, respectfully.

3. The early and late prosthesis survival rate in arches were implants had at least one misfit implant was $87.8 \%$ and $100 \%$, respectfully

4. Covariates such as arch (maxilla/mandible), implant position (anterior/posterior), type of implant (alveolar/extra maxillary), implant orientation (tilted/straight), connection (external/internal), surgery type (free-hand/guided) and total number of implants per arch played did not play a significant role in misfit $(\mathrm{p}>0.05)$.

The impact of misfit on implant survival in the immediate load scenario is minimal. However, this may not mean misfit can be forgiving. Misfit in interim CAFIPs can add an additional variable that may cause prosthesis complications. Overall the results of this study may 
aid clinician in achieve better clinical confidence and aid in decision making whether to correct a misfit if they experience a post-operative misfit in an immediately loaded CAFIP.

\section{FUTURE RESEARCH}

The use of dental implant therapy for complete arch rehabilitation will continue to gain popularity in the years to come. The results of the current study offer baseline survival data for practitioners to reference in the event they experience a misfit in the immediate load scenario. Future studies can expand on the baseline data provided by this study by exploring larger sample sizes and correlations between different co-variables. If available, future studies could use different modes of radiographic examination such as standardized periapical radiographs. Studies that offer a prospective analysis on this topic will also be beneficial to the dental community. 


\section{REFERENCES}

1. Schnitman P, Wöhrle P, Rubenstein J, DaSilva J, Wang N. Ten-year results for Brånemark implants immediately loaded with fixed prostheses at implant placement. Int J Oral Maxillofac Implants 1997;12: 495-503.

2. Balshi TJ, Wolfinger GJ. Conversion prosthesis: a transitional fixed implant-supported prosthesis for an edentulous arch--a technical note. Int J Oral Maxillofac Implants 1996; 11:106-11.

3. Maló P, Rangert B, Nobre M: "All-on-4” immeditate-fuction concept with Brånemark system implants for completely edentulous mandibles: a retrospective clinical study. Clin Implant Dent Relat Res 2003;5 Suppl 1:2-9.

4. Maló P, Rangert B, Nobre M: “All-on-4” immediate-function concept of Brånemark system implants for completely edentulous maxilla: a 1-year retrospective clinical study. Clin Implant Dent Relat Res 2005;7 Supp11:S88-S94

5. Papaspyridakos P, Mokti M, Chen CJ, Benic GI, Gallucci GO, Chronopoulos V. Implant and prosthodontic survival rates with implant fixed complete dental prostheses in the edentulous mandible after at least 5 years: a systematic review. Clin Implant Dent Relat Res $2014 ; 16: 705-17$.

6. Lambert FE, Weber HP, Susarla SM, Belser UC, Gallucci GO. Descriptive analysis of implant and prosthodontic survival rates with fixed implant-supported rehabilitations in the edentulous maxilla. J Periodontol 2009; 80:1220-30.

7. Mozzati M, Gallesio G, Del Fabbro M. Long-Term (9-12 Years) Outcomes of Titanium Implants with an Oxidized Surface: A Retrospective Investigation on 209 Implants. J Oral Implantol 2015; 41:437-43. 
8. Vigolo P, Mutinelli S, Zaccaria M, et al. Clinical evaluation of marginal bone level change around multiple adjacent implants restored with splinted and nonsplinted restorations: a 10-year randomized controlled trial. Int J Oral Maxillofac Implants $2015 ; 30: 411-418$.

9. Kan JY, Rungcharassaeng K, Bohsali K, Goodacre CJ, Lang BR. Clinical methods for evaluating implant framework fit. J Prosthet Dent 1999; 81:7-13.

10. Sahin S, Cehreli MC. The significance of passive framework fit in implant prosthodontics: current status. Implant Dent 2001;10:85-92.

11. Branemark PI. Osseointegration and its experimental background. J Prosthet Dent1983; 50:399-410.

12. Laney WR. Glossary of Oral and Maxillofacial Implants. Chicago: Quintessence Publishing Co 2007; 40,102,113,133.

13. Bidra AS, Taylor TD. Occlusion: the Role in Implant Prosthodontics; In: Sadowsky SJ (Ed), Evidence-based Implant Treatment Planning and Clinical Protocols, pp 169-184, Wiley, 2016.

14. Jemt T. Failures and complications in 391 consecutively inserted fixed prostheses supported by Branemark implants in edentulous jaws: a study of treatment from the time of prosthesis placement to the first annual checkup. Int J Oral Maxilofac Implants 1991; 6:270-6.

15. Drago C. Frequency and Type of Prosthetic Complications Associated with Interim, Immediately Loaded Full-Arch Prostheses: A 2-Year Retrospective Chart Review. J Prosthodont 2016; 25:433-9.

16. Jemt T, Book K. Prosthesis misfit and marginal bone loss in edentulous 
implant patients. Int J Oral Maxillofac Implants 1996; 11(5):620-625.

17. Nikolaus A, Currey JD, Lindtner T, Fleck C, Zaslansky P. Importance of the variable periodontal ligament geometry for whole tooth mechanical function: A validated numerical study. J Mech Behav Biomed Mater 2016: 25,67:61-73.

18. Katsoulis J, Mericske-Stern R, Yates DM, Izutani N, Enkling N, Blatz MB. In vitro precision of fit of computer-aided design and computer-aided manufacturing titanium and zirconium dioxide bars. Dent Mater 2013; 29:945-53.

19. Sharkey S, Kelly A, Houston F, O'Sullivan M, Quinn F, O'Connell B. A radiographic analysis of implant component misfit. Int J Oral Maxillofac Implants 2011; 26:807-15.

20. Papavassiliou H, Kourtis S, Katerelou J, Chronopoulos V. Radiographical evaluation of the gap at the implant-abutment interface. J Esthet Restor Dent. 2010; 22:235-50.

21. Lin KC, Wadhwani CP, Cheng J, Sharma A, Finzen F. Assessing fit at the implantabutment junction with a radiographic device that does not require access to the implant body. J Prosthet Dent 2014; 112:817-23.

22. Kan J, Rungcharassaeng K, Bohsali K, Goodacre C, Lang B. Clinical methods for evaluating implant framework fit. J Prosthet Dent 1999; 81:7-13.

23. Mattingly SL, Podshadley AG, Gullett CE. The use of radiographs in small cast-gold restorations. J Prosthet Dent 1982; 48:44-7.

24. Hämmerle CH. Success and failure of fixed bridgework. Periodontol 2000 1994; 4:41-51

25. Molander B. Panoramic radiography in dental diagnostics. Swed Dent J Suppl 1996; 119:1-26.

26. Karl M, Taylor TD, Wichmann MG, et al. In vivo stress behavior in cemented and screwretained five-unit implant FPDs. J Prosthodont 2006; 15: 20-24. 
27. Guichet DL, Caputo AA, Choi H, et al. Passivity of fit and marginal opening in screw- or cement-retained implant fixed partial denture designs. Int J Oral Maxillofac Implants $2000 ; 15: 239-246$.

28. Misch CE. Contemporary implant densitry. $3^{\text {rd }}$ ed. St. Lousi: Mosby; 2008. P 296-7.

29. Phillips K, Wong KM. Vertical space requirement for fixed-detechable, implantsupported prosthesis. Compend Cont Educ Dent 2002; 23:760-6.

30. Landázuri-Del Barrio R, Cosyn J, De Paula W, De Bruyn H, Marcantonio E. A prospective study on implants installed with flapless-guided surgery using the all-on-four concept in the mandible. Clin Oral Implants Res 2013; 24: 428-33.

31. Yilmaz B, Suarez C, McGlumphy E. Correction of misfit in a maxillary immediate metalresin implant-fixed complete prosthesis placed with flapless surgery on four implants. Int J Oral Maxillofac Implants 2011; 26: 23-8.

32. Oyama, Kan J, Kleinman A, Runcharassaeng K, Lozada J, Goodacre C. Misfit of implant fixed complete denture following computer-guided surgery. Int J Oral Maxillofac Implants 2009; 24:124-30.

33. Brånemark PI, Adell, R, Breine, U, Hansson B, Lindström, J. \& Ohlsson, A. Intraosseus anchorage of dental prostheses. 1. Experimental studies. Scand J Plast Reconstr Surg 1969; 3: 81-100.

34. Brånemark PI, Hansson, B. \& Adell, R. Osseointegrated implants in the treatment of the edentulous jaw. Experience from a 10-year period. Scand J Plast Reconstr Surg 1977; 16 : $1-32$. 
35. SchroederA, Van Den Zypen E, Stich H, Sutter F. The reaction of bone, connective tissue and epithelium to endosteal implants with titanium-sprayed surfaces. J Maxillofac Surg 1981; 9: 15-25.

36. Attard N, Zarb G. Immediate and early implant loading protocols: a literature review of clinical studies. J Prosthet Dent 2005; 94: 242-58.

37. Sennerby L, Meredith N. Implant stability measurements using resonance frequency analysis: biological and biomechanical aspects and clinical implications. Periodontol $20002008 ; 47: 51-66$.

38. Tirachaimongkol C, Pothacharoen P, Reichart PA, Khongkhunthian P. Relation between the stability of dental implants and two biological markers during the healing period: a prospective clinical study. Int J Implant Dent 2016; 2:27.

39. Balshi SF, Allen FD, Wolfinger GJ, Balshi TJ. A resonance frequency analysis assessment of maxillary and mandibular immediately loaded implants. Int J Oral Maxillofac Implants 2005; 20:584-94.

40. Balshi TJ, Wolfinger GJ, Slauch RW, Balshi SF. A retrospective analysis of 800 Brånemark System implants following the All-on-Four protocol. J Prosthodont 2014; 23:83-8.

41. Niedermaier R, Stelzle F, Riemann M, Bolz W, Schuh P, Wachtel H. Implant-Supported Immediately Loaded Fixed Full-Arch Dentures: Evaluation of Implant Survival Rates in a Case Cohort of up to 7 Years. Clin Implant Dent Relat Res 2016; 15:1-11.

42. Douglass CW, Shih A, Ostry L. Will There be a Need for Complete Dentures in the United States in 2020? J Prosthet Dent 2002; 87:5-8. 
43. Manzella C, Bignardi C, Burello V, Carossa S, Schierano G. Method to improve passive fit of frameworks on implant-supported prostheses: An in vitro study. J Prosthet Dent $2016 ; 116: 52-8$

44. Abduo J, Lyons K. Effect of misfit on strain within screw-retained implant titanium and zirconia frameworks. J Prosthodont Res 2012; 56:102-9.

45. Papaspyridakos P, Chen CJ, Chuang SK, Weber HP. Implant loading protocols for edentulous patients with fixed prostheses: a systematic review and metaanalysis. Int J Oral Maxillofac Implants. 2014; 29 Suppl:256-70.

46. Drago C. Cantilever Lengths and Anterior-Posterior Spreads of Interim, Acrylic Resin, Full-Arch Screw-Retained Prostheses and Their Relationship to Prosthetic Complications. J Prosthodont. 2017 Aug;26(6):502-507.

47. Bidra AS. Technique for systematic bone reduction for fixed implantsupported prosthesis in the edentulous maxilla. J Prosthet Dent. 2015; 113(6):520-3. 


\section{FIGURES AND TABLES}

Table 1: Summary of implants and interim prostheses analyzed in this retrospective study to study misfit in the immediate load scenario.

\begin{tabular}{|l|l|}
\hline Total number of implants screened & 2025 \\
\hline Total number of arches screened & 425 \\
\hline Total number of patient charts screened & 311 \\
\hline Age range of patients screened & $32-89$ yrs. \\
\hline Total number of implants associated with radiographic misfit & 48 \\
\hline Total number of arches associated with radiographic misfit & 33 \\
\hline Total number of patients associated with radiographic misfit & 27 \\
\hline $\begin{array}{l}\text { Total number of implant failures } \\
\text { Total number of arches associated with misfit and prosthesis complications }\end{array}$ & 2 \\
\hline Total number of patients associated with misfit and prosthesis complications & 4 \\
\hline Number of implants in the arches where misfit occurred* & 182 \\
\hline Misfit implants* & 48 \\
\hline Non-Misfit implants * & 134 \\
\hline
\end{tabular}

* Refer to Table 2 
Table 2: Prevalence of demographic and clinical covariates in the combined 182 implants (misfit and non-misfit) in arches where misfit occurred.

\begin{tabular}{|c|c|c|c|c|}
\hline & $\begin{array}{l}\text { Misfit } \\
(\mathrm{n}=48)\end{array}$ & $\begin{array}{l}\text { Non-Misfit } \\
(\mathrm{n}=134)\end{array}$ & $\begin{array}{l}\text { Combined } \\
(\mathrm{n}=182)\end{array}$ & P-value \\
\hline Arch & & & & 0.876 \\
\hline Mandible & $12(25 \%)$ & $32(24 \%)$ & $44(24 \%)$ & \\
\hline Maxilla & $36(75 \%)$ & $102(76 \%)$ & $138(76 \%)$ & \\
\hline Gender & & & & 0.798 \\
\hline Female & $28(58 \%)$ & $81(60 \%)$ & $109(60 \%)$ & \\
\hline Male & $20(42 \%)$ & $53(40 \%)$ & $73(40 \%)$ & \\
\hline \multicolumn{5}{|l|}{ Misfit Present } \\
\hline No & $0(0 \%)$ & $134(100 \%)$ & $134(74 \%)$ & \\
\hline Yes & $48(100 \%)$ & $0(0 \%)$ & $48(26 \%)$ & \\
\hline Surgery Type & & & & 0.520 \\
\hline Free Hand & $42(88 \%)$ & $112(84 \%)$ & $154(85 \%)$ & \\
\hline Guided & $6(12 \%)$ & $22(16 \%)$ & $28(15 \%)$ & \\
\hline Misfit Implant Position & & & & 0.549 \\
\hline Anterior & $21(44 \%)$ & $52(39 \%)$ & $73(40 \%)$ & \\
\hline Posterior & $27(56 \%)$ & $82(61 \%)$ & $109(60 \%)$ & \\
\hline Implant Location & & & & 0.897 \\
\hline Alveolar & $35(73 \%)$ & $99(74 \%)$ & $134(74 \%)$ & \\
\hline Extra-maxillary & $13(27 \%)$ & $35(26 \%)$ & $48(26 \%)$ & \\
\hline \multicolumn{5}{|l|}{ Implant Orientation } \\
\hline Straight & $21(44 \%)$ & $56(42 \%)$ & $77(42 \%)$ & \\
\hline Tilted & $27(56 \%)$ & $78(58 \%)$ & $105(58 \%)$ & \\
\hline Total \# Implants (per Arch) & $5.6 \pm 1.61$ & $5.81 \pm 1.58$ & $5.76 \pm 1.58$ & 0.431 \\
\hline Implant-Abutment Connection & & & & 0.818 \\
\hline External & $31(65 \%)$ & $89(66 \%)$ & $120(66 \%)$ & \\
\hline Internal & $17(35 \%)$ & $45(34 \%)$ & $62(34 \%)$ & \\
\hline Misfit Location & & & & 0.156 \\
\hline Abutment Level & $46(96 \%)$ & $133(99 \%)$ & $179(98 \%)$ & \\
\hline Implant Level & $2(4 \%)$ & $1(1 \%)$ & $3(2 \%)$ & \\
\hline \multicolumn{5}{|l|}{ Misfit Resolution? } \\
\hline Yes & $46(96 \%)$ & $2(2 \%)$ & $48(26 \%)$ & \\
\hline N/A & $2(4 \%)$ & $132(98 \%)$ & $134(74 \%)$ & \\
\hline \multicolumn{5}{|l|}{ Implant Failure? } \\
\hline No & $46(96 \%)$ & $134(100 \%)$ & $180(99 \%)$ & \\
\hline Yes & $2(4 \%)$ & $0(0 \%)$ & $2(1 \%)$ & \\
\hline Prosthesis Failure? & & & & 0.375 \\
\hline
\end{tabular}




\begin{tabular}{|l|l|l|l|l|}
\hline No & $43(90 \%)$ & $113(84 \%)$ & $156(86 \%)$ & \\
\hline Yes & $5(10 \%)$ & $21(16 \%)$ & $26(14 \%)$ & \\
\hline
\end{tabular}

N/A: Implant did not resolve due to failure 
Figure 1: Immediate post-operative panoramic radiograph demonstrating vertical misfit at the abutment-prosthesis interface at site no. 13

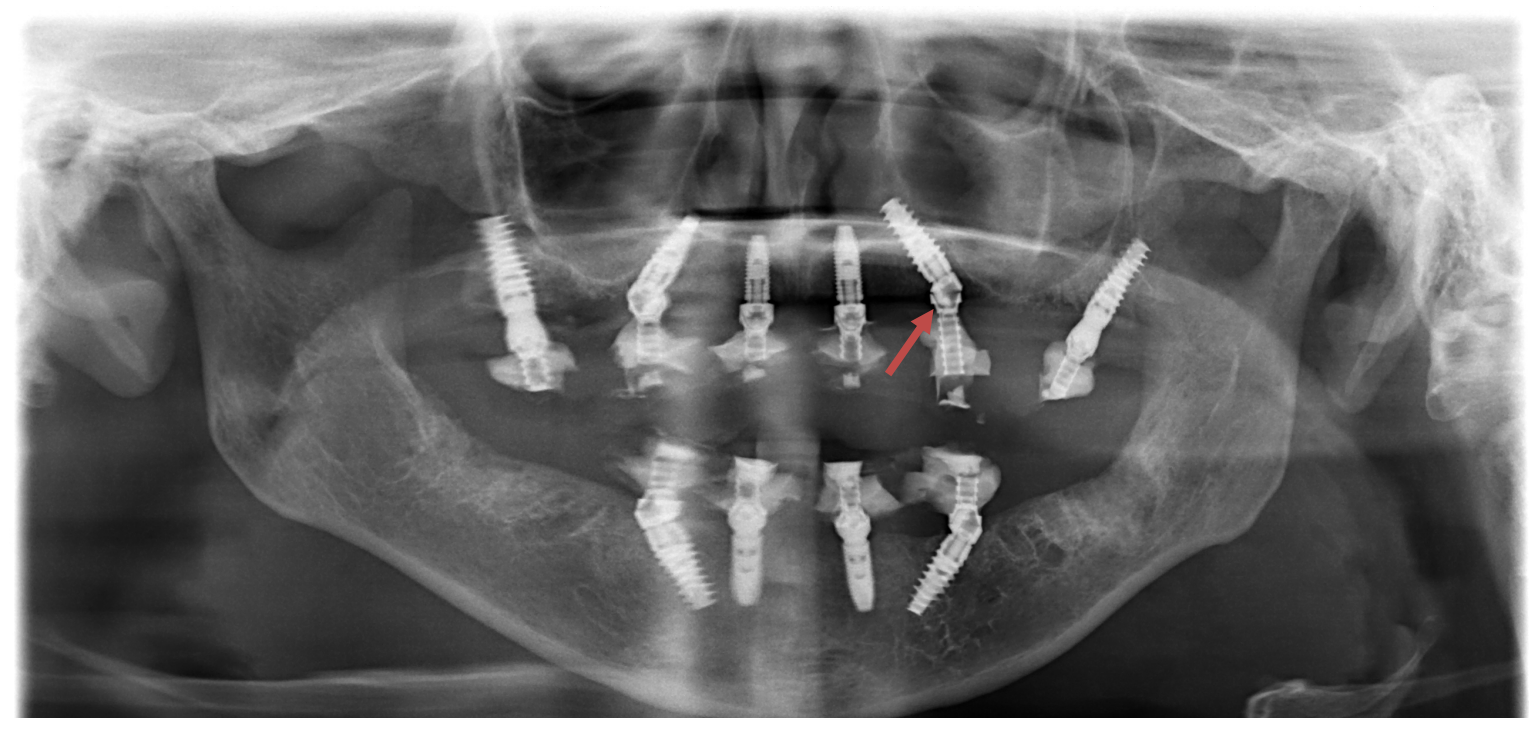


Figure 2: Three-to-four-month follow-up panoramic radiograph demonstrating resolution of misfit at the same interface.

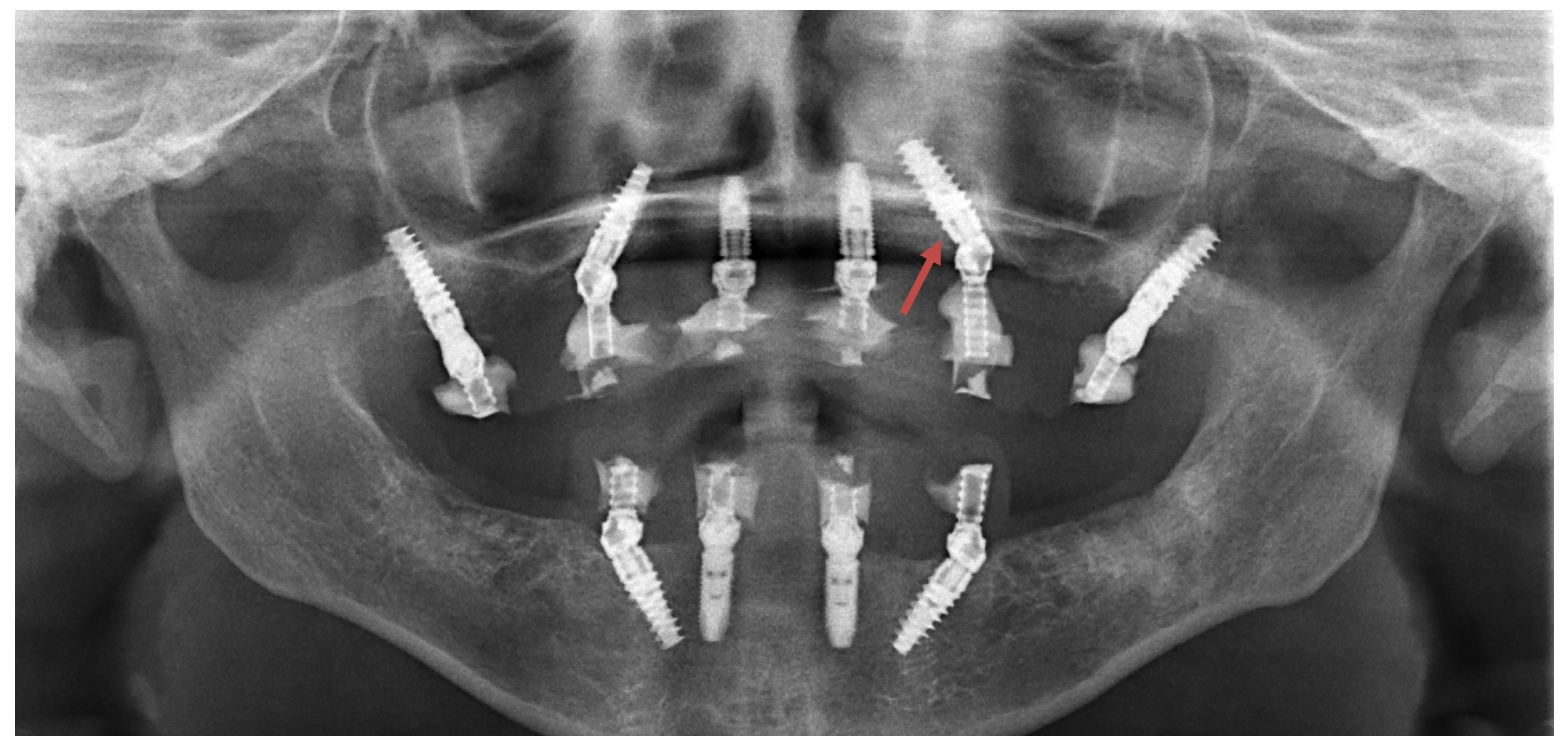


Figure 3: Post-insertion panoramic radiograph of the definitive prosthesis.

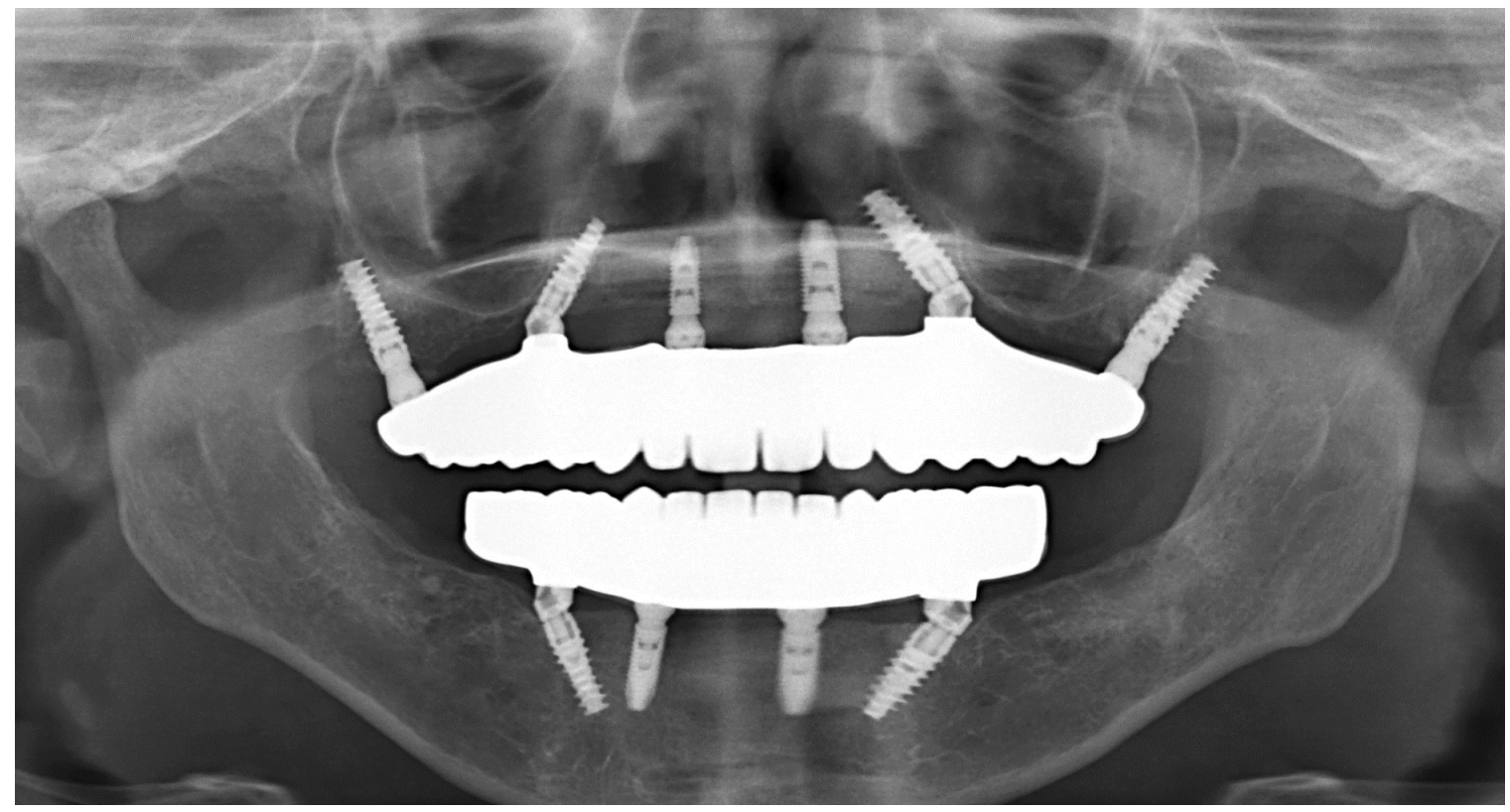


Figure 4: Implant-abutment misfit in the immediate load scenario. prosthesis.

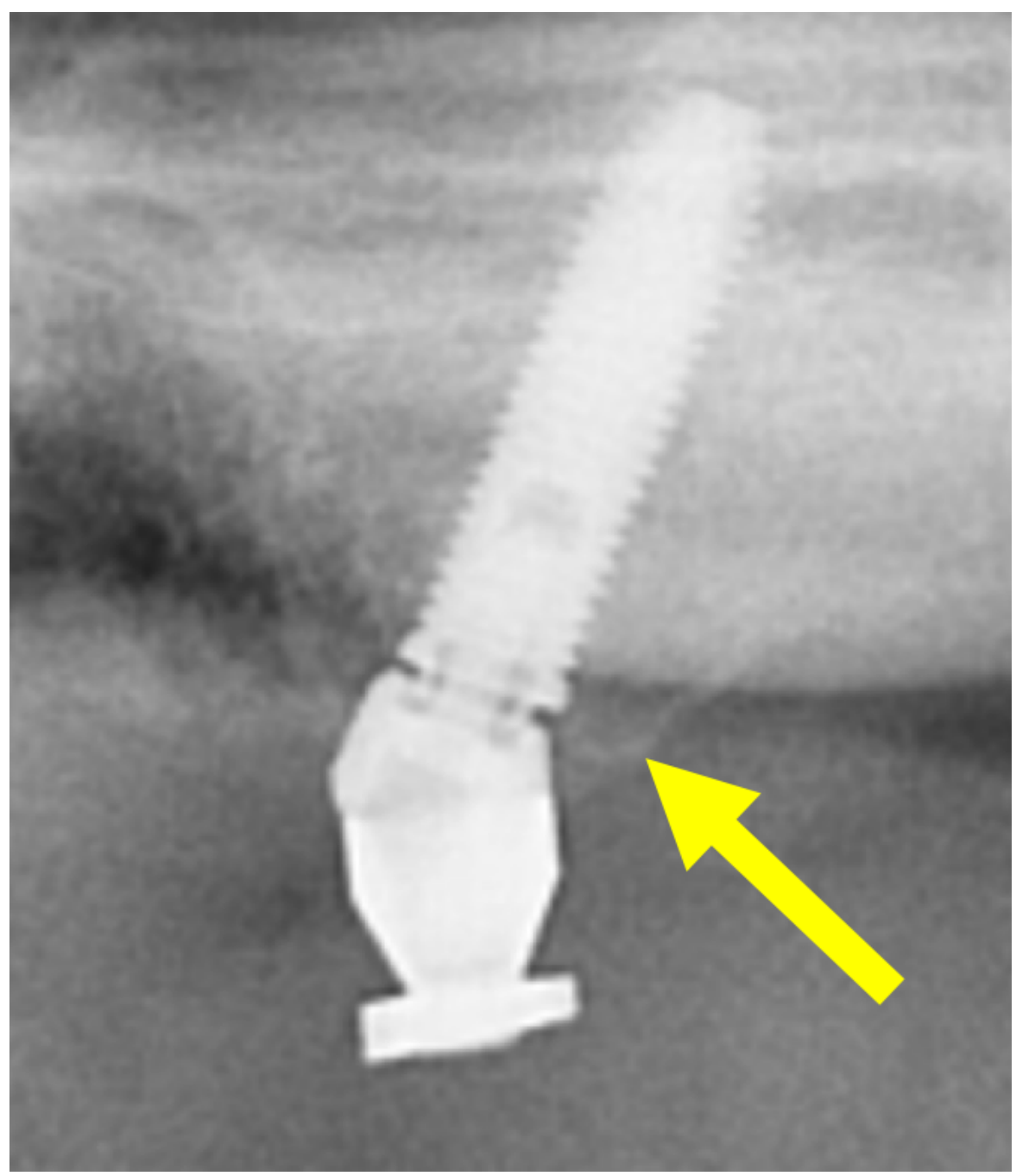


Figure 5: Implant-prosthesis misfit in the immediate load scenario.

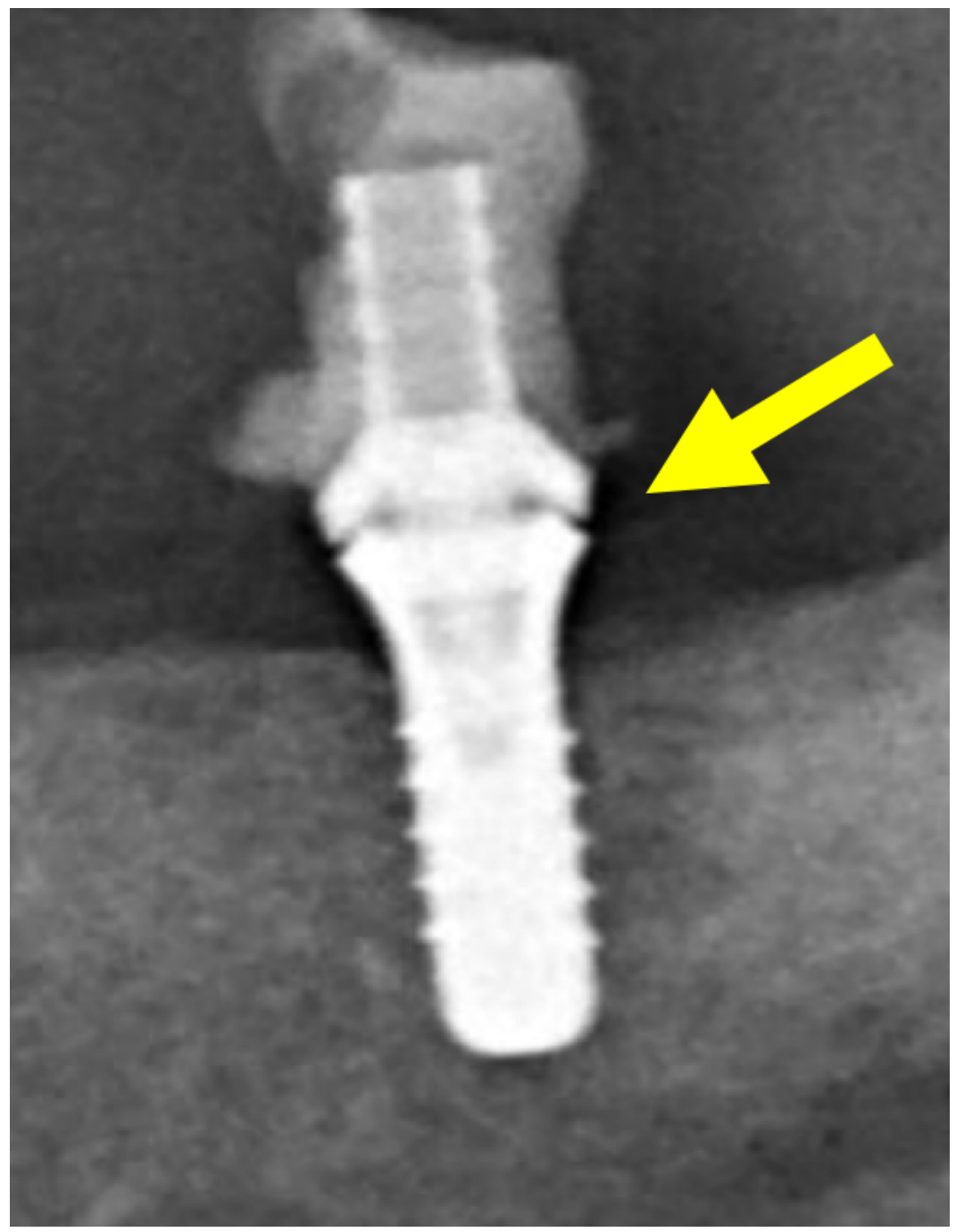


Figure 6: Abutment-temporary coping misfit in the immediate load scenario.

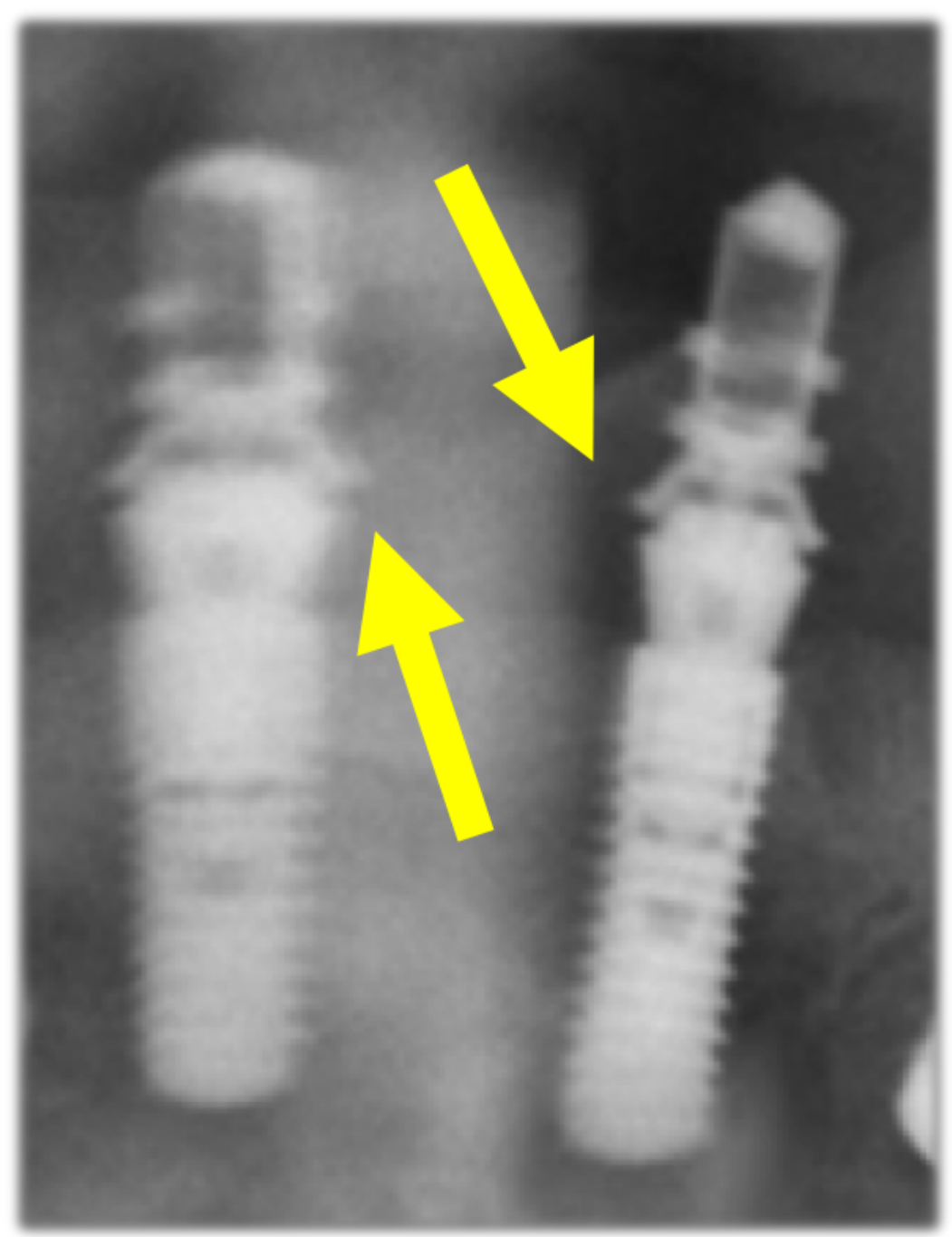


Figure 7: Prevalence of radiographic detectible misfit in the maxilla and mandible

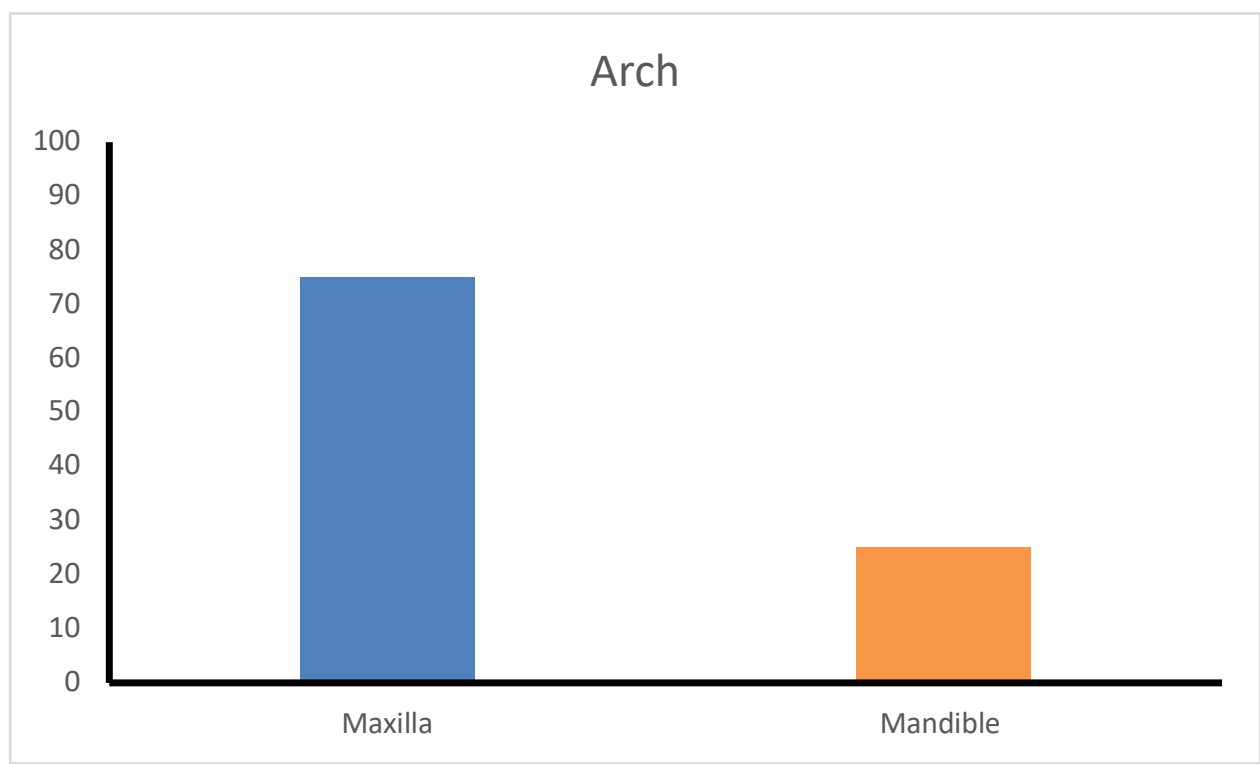


Figure 8: Prevalence of radiographic detectible misfit in the anterior and posterior implant positions.

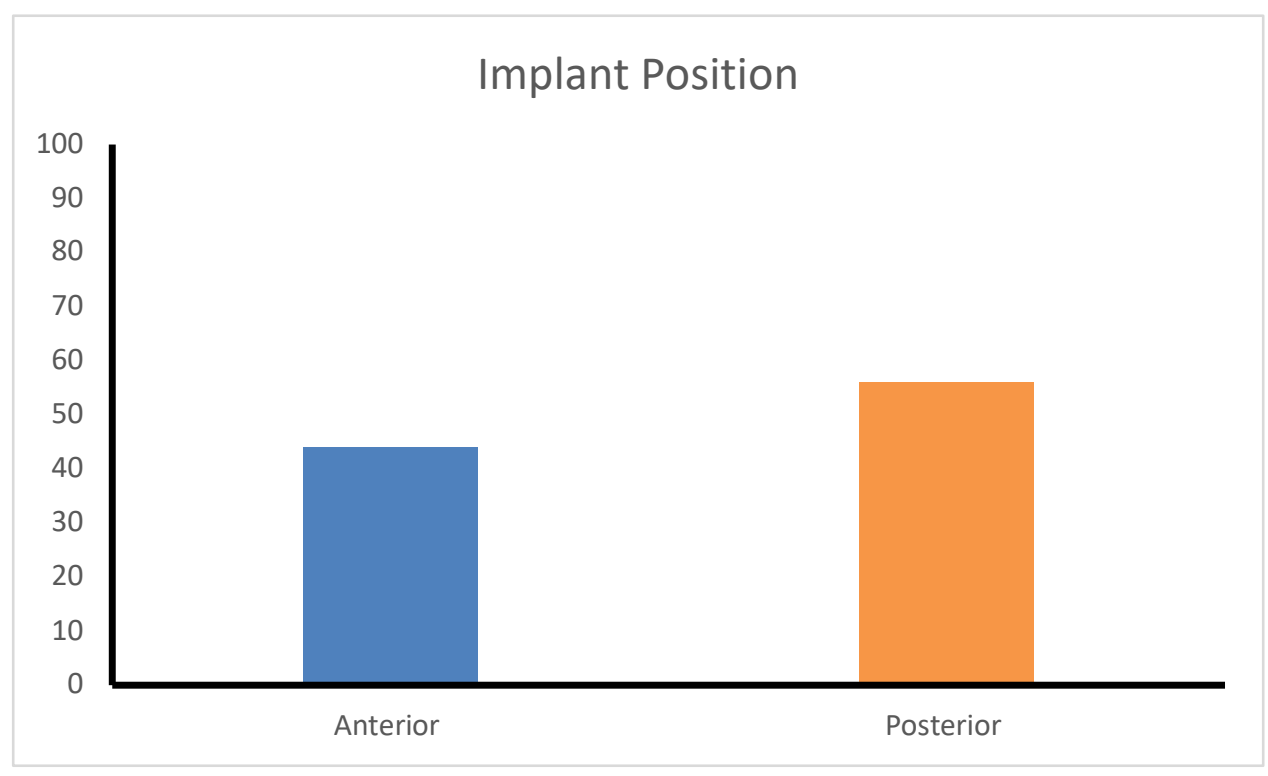


Figure 9: Prevalence of radiographic detectible misfit in the type of implant placed.

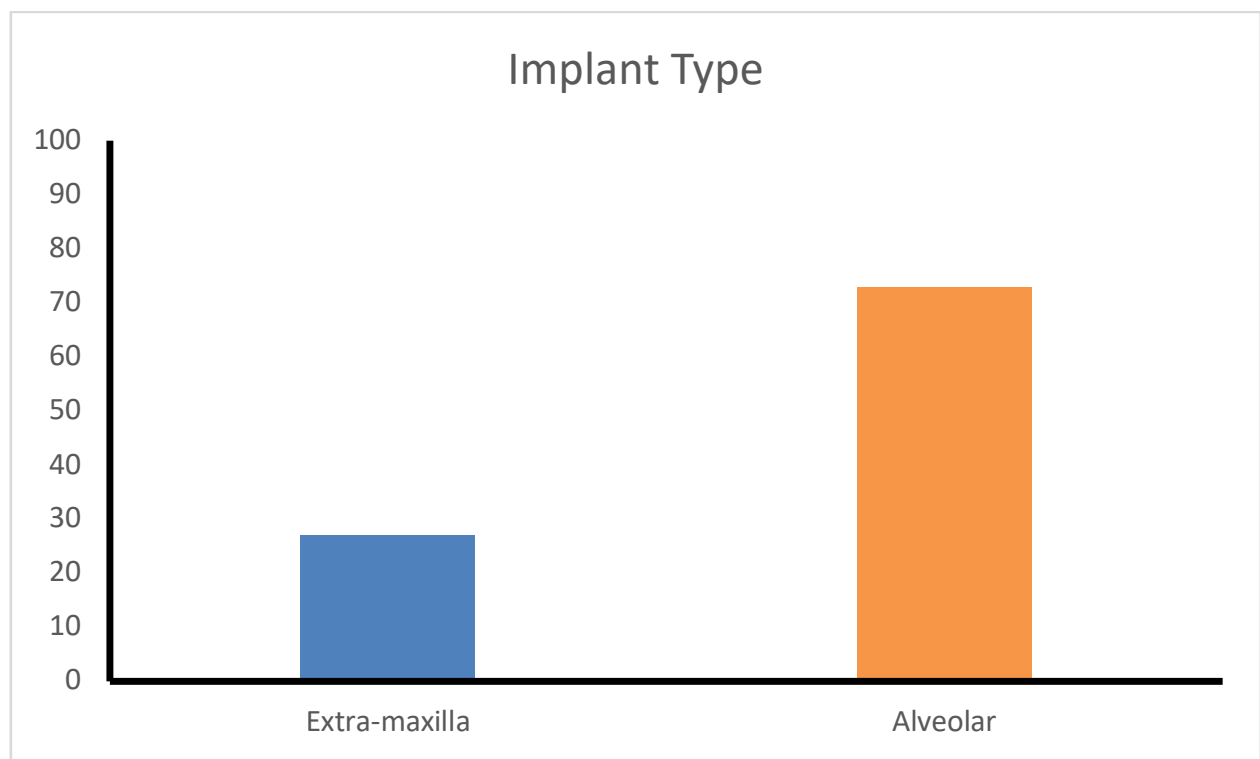

Extra-maxilla: Pterygomaxillary and/or zygomatic implants 
Figure 10: Prevalence of radiographic detectible misfit with type of implant orientation.

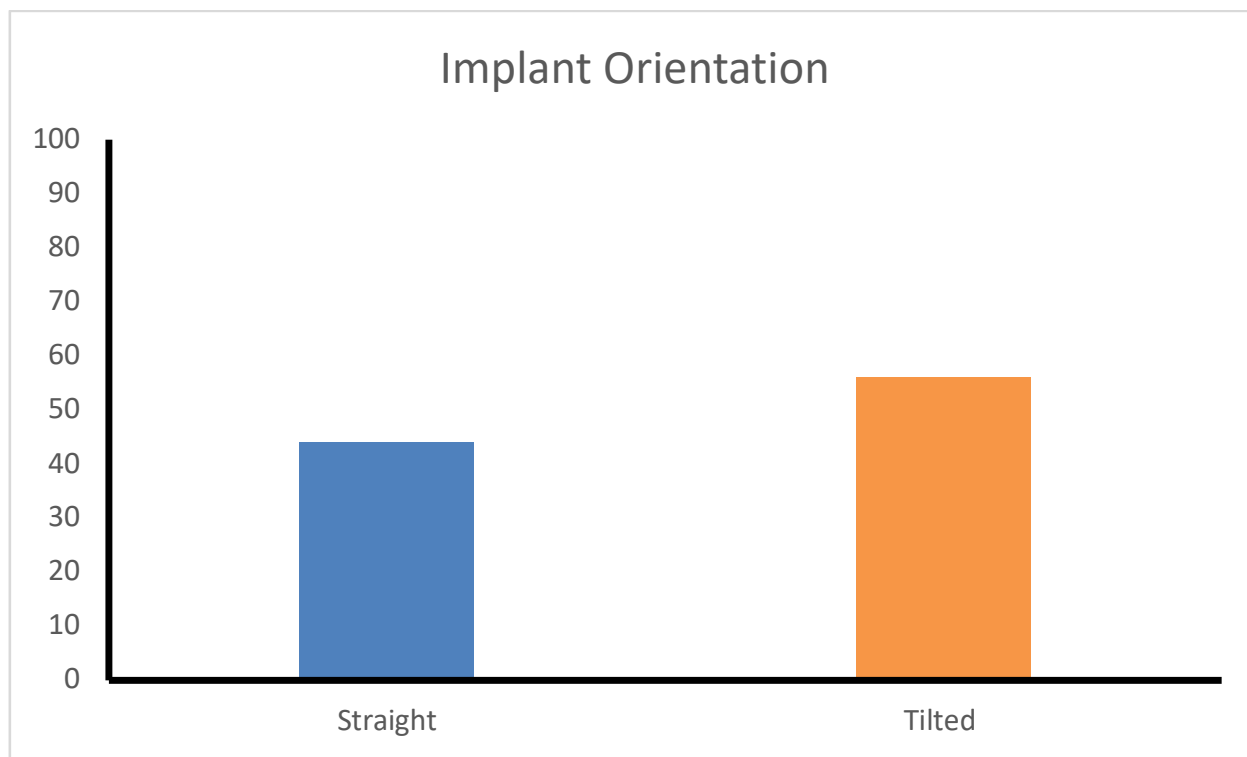


Figure 11: Prevalence of radiographic detectible misfit with type of implant-abutment connection.

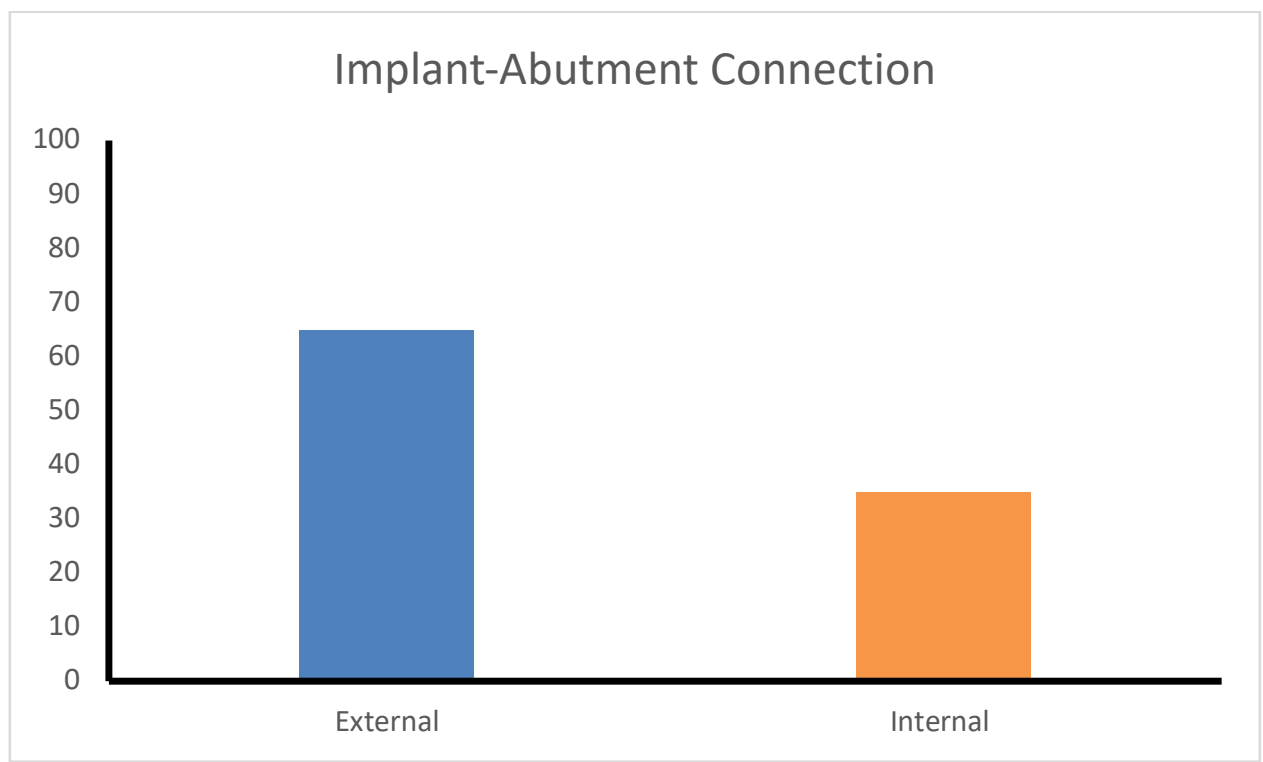


Figure 12: Prevalence of radiographic detectible misfit in the type of implant surgery performed.

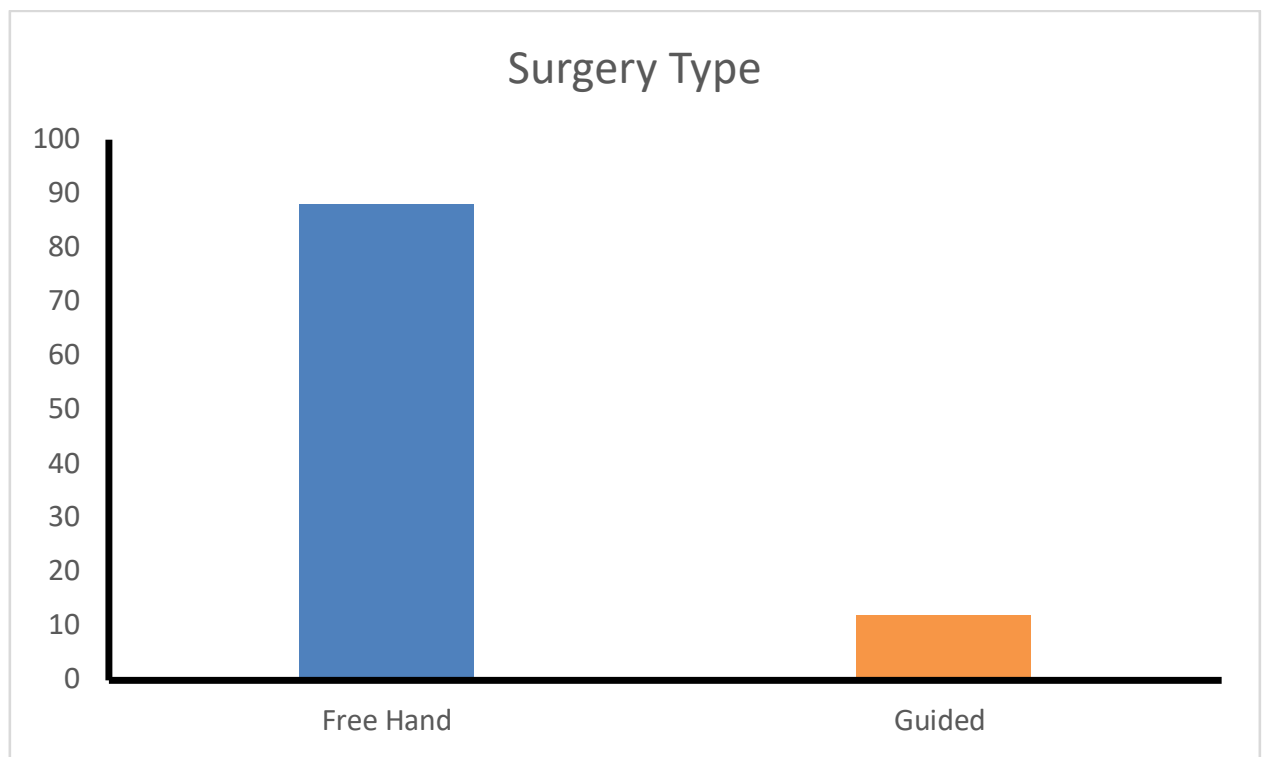

